age at baseline $=56 ; 55 \%$ female). Risk factors will be determined at baseline (from 2006 to 2010), and COVID-19 outcomes will be ascertained from linked Public Health England COVID-19 test data and mortality statistics.Univariate cox proportional hazard models will be used to explore how associations between time-varying and time-stable variables change over time with; i) having a test for COVID-19, ii) testing positive for COVID-19 and iii) dying with COVID19. Time-varying risk factors will be based on measures of socioeconomic position (SEP) including education, Townsend deprivation index and income. $\mathrm{ABO}$ blood group will be considered as a time-stable risk factor. Distinct time periods will be defined based on changes in testing definitions and changes in lockdown restrictions. Inverse probability weights will then be calculated for each time period. These weights will then be applied to models estimating risk across all time periods.

Expected Results Preliminary analyses show that the size of the association between SEP and i) COVID-19 testing and ii) testing positive for COVID-19, changes across the course of the pandemic. These differences may be due to differential testing and not time-varying causal effects of the risk factor. We expect inverse probability weights will provide estimates closer to the true value for the association between each risk factor and outcome, independent of selection pressures on receiving a COVID-19 test. Population Health Relevance. Where studies do not account for timevarying selection pressures, the causal interpretations and the validity of results may be distorted. Where these findings are to be translated into developing population level or pharmaceutical interventions to mitigate against COVID-19 outcomes, efforts may be diverted away from more important risk factors.

\section{P51 A SURVEY OF THE IMPACT OF WAVE ONE OF THE COVID-19 PANDEMIC ON PARTICIPANTS OF THE HERTFORDSHIRE COHORT STUDY}

Gregorio Bevilacqua, Alice Paul, Ilse Bloom*, Karen Jameson, Jean Zhang, Cyrus Cooper, Kate Ward, Elaine Dennison. MRC Lifecourse Epidemiology Unit, University of Southampton, Southampton, UK

\subsection{6/jech-2021-SSMabstracts. 139}

Background Covid-19, a coronavirus that originated in China in late 2019, spread globally to be declared a pandemic by the World Health Organisation in March 2020. The aim of this study was to usean existing cohort of community-dwelling older adults, the Hertfordshire Cohort Study (HCS), to understand how wave one of the Covid-19 pandemic impacted UK older adults, a group particularly vulnerable to severe disease.

Methods 71 eligible participants, 39 males and 32 females (drawn from the HCS study, mean age 83.6 (2.5) years, all Caucasian, and community dwelling) were contacted by telephone and asked to complete a questionnaire administered by a trained researcher. Data collection occurred over the period of July to October 2020.

Results Over a third (37.1\%) of respondents lived alone. Of the remainder, $86.4 \%$ lived with a spouse while the remaining $13.6 \%$ lived with family. Of concern, $19.7 \%$ of participants had felt they needed to go out despite not wanting to; $47.1 \%$ had heard of the NHS Volunteer Responders programme, although only $3(4.2 \%)$ had made use of this or other support services. Almost a third (31\%) of participants reported they had access to a smartphone, while $62 \%$ reported having unlimited internet access, usually using a tablet or computer. Over two thirds (69.0\%) of participants rated their understanding of Covid-19 itself as good. Perhaps unsurprisingly, a large majority $(88.7 \%)$ of participants said their life was different compared to before Covid-19; $80.3 \%$ had less social contact and more than half (52\%) of respondents reported being less physically active than before the pandemic (and only $4 \%$ more so). However, levels of sleep, alcohol consumption and diet were reportedly generally unchanged over the timeframe surveyed.

Conclusion We have reported the experience of the first wave of the Covid-19 pandemic among participants of an older Caucasian community-dwelling UK cohort, highlighting the need to consider this group when developing public health interventions to support health and wellbeing, including those employing smartphone technology. Further larger studies in groups of wider socioeconomic status and more diverse racial background are indicated.

\section{P52 1 THE EXPERIENCES OF COMMUNITY-DWELLING OLDER ADULTS DURING THE COVID-19 PANDEMIC: PRELIMINARY FINDINGS FROM A QUALITATIVE STUDY WITH HERTFORDSHIRE COHORT STUDY PARTICIPANTS}

\footnotetext{
1,2 Ilse Bloom*, 1,2 Jean Zhang, 'Gregorio Bevilacqua, 1,2Wendy Lawrence, 'Kate Ward ${ }^{1}$ Cyrus Cooper, ${ }^{1}$ Elaine Dennison. ${ }^{1} M R C$ Lifecourse Epidemiology Unit, University of Southampton, Southampton, UK; ${ }^{2}$ NIHR Southampton Biomedical Research Centre, University of Southampton and University Hospital Southampton NHS Foundation, Southampton, UK
}

\subsection{6/jech-2021-SSMabstracts. 140}

Background The COVID-19 pandemic has led to dramatic changes in people's lives globally. Older adults have been especially vulnerable to adverse effects from the pandemic including higher mortality and more severe disease complications. At the same time, social isolation, malnutrition and physical inactivity are serious concerns among older adults. The pandemic and associated restrictions may serve to exacerbate these issues, presenting increased risks to physical and mental health. The aim of this qualitative study was to explore how community-living older people in the UK experienced the first wave of the COVID-19 pandemic and how it impacted their health and well-being, and associated behaviours.

Methods Qualitative data were collected by conducting serial telephone interviews, with an interval of approximately three months. Participants were from the Hertfordshire Cohort Study, all aged over 80 years. Discussions were audiorecorded, information related to the COVID-19 pandemic was transcribed verbatim and transcripts analysed thematically. Interviews were conducted from March to October 2020.

Results Twelve participants were included in the study (7 men and 5 women). Data from a total of 35 qualitative interviews were used, comprising two or three timepoints per participant. Preliminary analysis identified five overarching themes: 1) shopping strategies and food accessibility, 2) limitations on activities and going out, 3) disruption to healthcare, 4) social and psychological repercussions, and 5) coping strategies. Initial findings highlight challenges associated with accessing 
shops, healthcare, and usual activities due to restrictions. Findings emphasize the issue of loneliness and isolation for some of the participants, especially those living alone, as well as fear of the virus, with restrictions leading to a loss of purpose for some, along with related effects on mental health (e.g. worry, anxiety). For some, these issues appeared to link to a reprioritisation of their behaviours (for example, exercise and diet were deprioritised). Coping strategies used by participants included finding ways to keep busy and to do physical activity safely, maintaining social contact online or by telephone, and having an optimistic or positive outlook, a 'do what you can' attitude.

Conclusion Analysis is ongoing and will further aim to explore how older people's experiences and behaviours might have changed over the duration of the pandemic. The findings from this study could improve understanding of how community-living older adults could be supported to be more resilient in the face of a variety of changing circumstances that might impact their health and well-being.

\section{P53 EFFECTS OF COVID-19 PUBLIC HEALTH SAFETY MEASURES ON BIRTHS IN SCOTLAND BETWEEN MARCH AND MAY 2020}

${ }^{1}$ Louise Marryat*, ${ }^{2}$ Lydia Speyer, ${ }^{2}$ Bonnie Auyeung. ${ }^{1}$ School of Health Sciences, University of Dundee, Dundee, UK; ${ }^{2}$ School of Psychology, University of Edinburgh, Edinburgh, UK

\subsection{6/jech-2021-SSMabstracts. 141}

Background To combat the wide-spread transmission of COVID-19, many countries, including the Scotland, have imposed nationwide lockdowns. This included changes to NHS maternity and ante/postnatal services and policies. Little is known about how these lockdown measures affect pregnant mothers and their offspring. This study aimed to explore the impact of COVID19 public health safety measures on birth outcomes in Scotland.

Methods Using routinely collected health data on pregnancy and birth in Scotland, this study compares all births $(\mathrm{N}=$ 11220) between March and May 2020 to births in the same period in $2018(\mathrm{~N}=12428)$ to investigate the potential effects of lockdown measures introduced in Scotland in spring 2020. A variety of maternal and infant outcomes were analysed: induction of labour (yes, no), mode of delivery (unassisted vaginal delivery, planned caesarean section, emergency caesarean section, other (e.g. use of forceps)), analgesia during labour (none, gas and air, opioids, epidural, spinal anaesthetic, general anaesthetics, other), birth outcome (livebirth, stillbirth, infant death), APGAR score $(<3,4-<7,>7)$, age of gestation, birthweight, length of hospital stay, and feeding method on discharge (breastfeeding, formula, mixed feeding, other). Data were analysed using Mann-Whitney U tests for continuous outcomes and chi-square tests of independence for nominal outcomes. If the chi-square test was significant $(\alpha<0.05)$, post-hoc tests (Fisher's exact tests) were conducted to examine all possible comparisons. These were corrected for multiple comparisons using Bonferroni adjustment.

Results Overall there were few statistically significant differences between births in 2018 and 2020: women in 2020 were more likely to require spinal anaesthetics compared to using no pain relief air $(P=.035)$, gas and air $(P<.001)$ or opioids $(P<.001)$ as well as more likely to have an epidural compared to using gas and air $(P<.001)$ or opioids $(P<$ .001). Mothers giving birth during the pandemic also tended to combine breastfeeding and formula-feeding on discharge $\left(\chi^{2}(3)=19.09, p<.001\right)$ : post-hoc tests revealed that women were more likely to combine breastfeeding with formula-feeding than to exclusively breastfeed $(P<.001)$ or exclusively formula-feed $(P<.001)$. Finally, women stayed in hospital for fewer days $(Z=-10.90, p<.001)$ and more often had an epidural or a spinal anaesthetic compared to women giving birth in 2018.

Conclusion In conclusion, findings of the current study suggest that lockdown measures implemented in Scotland as a response to the COVID-19 pandemic had limited effects on maternal and neonatal outcomes. While these findings are reassuring, future research is needed to gain better insights into the impact of COVID-19 and associated lockdown measures on maternal and child health.

\section{P54 PHASE 2 OF THE NORWICH COVID-19 TESTING INITIATIVE: AN EVALUATION}

${ }^{1}$ Tara Berger Gillam*, ${ }^{2}$ Jeannette Chin, ${ }^{3}$ Karim Gharbi, ${ }^{5}$ Mark Hitchcock, ${ }^{4}$ Rose Davidson, ${ }^{3}$ Neil Shearer, ${ }^{1}$ Nick Steel. ${ }^{1}$ Health Services and Primary Care Research Group, University of East Anglia, Norwich, UK; ${ }^{2}$ School of Computing Sciences, University of East Anglia, Norwich, UK; ${ }^{3}$ Earlham Institute, Norwich, UK; ${ }^{4}$ Faculty of Medicine and Health Sciences, University of East Anglia, Norwich, UK; ${ }^{5}$ UEA Health and Social Care Partners, University of East Anglia, Norwich, UK

\subsection{6/jech-2021-SSMabstracts. 142}

Background Phase 2 of the Norwich Testing Initiative (NTI2) was a SARS-CoV-2 PCR testing programme at the University of East Anglia, which ran from September to December 2020. It aimed to identify asymptomatic COVID-19 infections and limit outbreaks on campus. The NTI2 evaluation explored testing uptake, positivity rates, isolation compliance and the links between viral load and symptoms.

Methods All staff and students $(21,762)$ were eligible for testing. Users registered for the programme using a web application, which collected personal, demographic and location data. Users collected tests from a central location on campus, selfadministered a nasopharyngeal swab and returned tests to a drop-off point. PCR testing was conducted by two laboratories. All those with a positive result were contacted by telephone for self-isolation advice and support. At this point symptom, isolation and location data were collected. The programme used a broader definition of COVID-19 symptoms than that used by NHS Test and Trace. Data were encrypted and stored in a data warehouse. Microsoft Excel was used to collate, clean and analyse the data.

Results 188 of 6537 (2.9\%) users tested positive for COVID19. The majority (82\%) were students aged 18-24 years. Positivity rates were highest in students $(3.5 \%)$, those living on campus (6.5\%) and BAME groups (4\%). There was clustering of cases in university halls: positivity rates in halls varied between $0 \%$ and $31 \%$, with $18 \%$ of halls containing over half the cases on campus. Positivity rates spiked near the beginning of the programme and then declined over the course of the term. 99/187 (53\%) cases reported symptoms of COVID-19. $37 / 99(35 \%)$ symptomatic cases were not isolating. There was no significant relationship between PCR $C_{t}$ values and selfreported symptoms.

Conclusion NTI2 may have contributed to a decline in cases of COVID-19 during the testing period, as the incidence of 